TecnoLógicas

ISSN 0123-7799

ISSN-e 2256-5337

Vol. 20, No. 39

Mayo - agosto de 2017

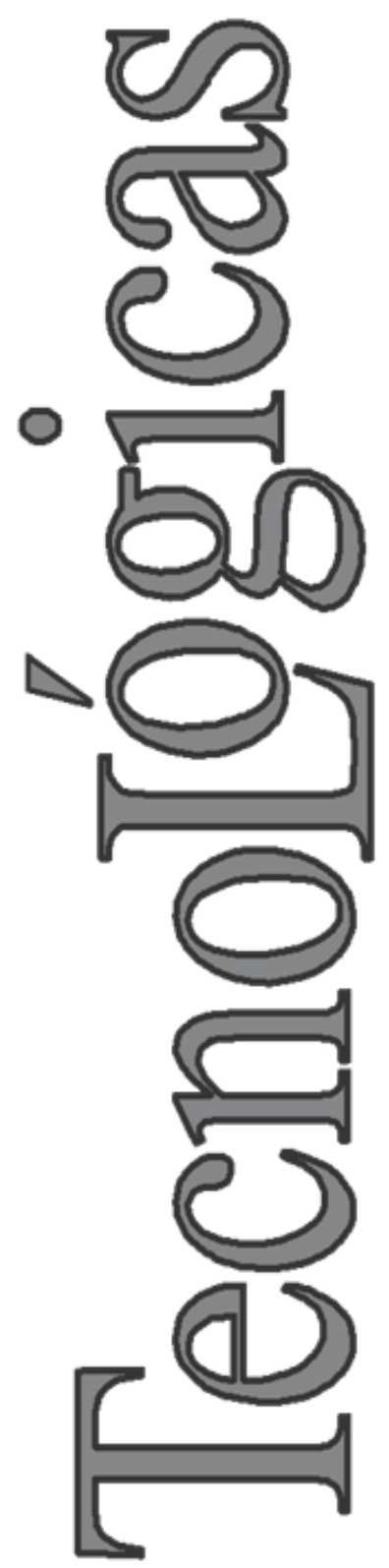

(C) Copyright 2015 por autores y Tecno Lógicas Este trabajo está licenciado bajo una Licencia Internacional Creative Commons Atribución (CC BY)

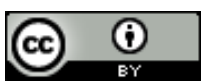

\section{Reconfiguración de paneles fotovoltaicos para reducción del consumo de hidrógeno en las celdas de combustible de sistemas híbridos}

\section{Reconfiguration of photovoltaic panels for reducing the hydrogen consumption in fuel cells of hybrid systems}

\author{
Daniel González-Montoya ${ }^{1}$, Carlos. A. Ramos-Paja², \\ Freddy Bolaños-Martínez ${ }^{3}$, Fabio Ramírez-Quiroz ${ }^{4}$, \\ Juan R. Camarillo-Peñaranda ${ }^{5}$ y Adriana Trejos- \\ Grisales ${ }^{6}$
}

Recibido: 04 de agosto de 2016, Aceptado: 15 de marzo de 2017

Cómo citar / How to cite

D. González-Montoya, C.A. Ramos-Paja, F. Bolaños-Martínez, F. Ramírez-Quiroz, J.R. Camarillo-Peñaranda y A. Trejos-Grisales, "Reconfiguración de paneles fotovoltaicos para reducción del consumo de hidrógeno en las celdas de combustible de sistemas híbridos", TecnoLógicas, vol. 20, no. 39, 2017.

1 MSc en Ingeniería Eléctrica, Departamento de Electrónica y Telecomunicaciones, Facultad de Ingenierías, Instituto Tecnológico Metropolitano, Medellín-Colombia, danielgonzalez@itm.edu.co

$2 \mathrm{PhD}$ Ingeniería Electrónica, Automática y Comunicaciones, Departamento de Energía Eléctrica y Automática, Universidad Nacional de Colombia, Medellín-Colombia, caramosp@unal.edu.co

3 PhD Ingeniería Electrónica, Departamento de Energía Eléctrica y Automática, Universidad Nacional de Colombia, MedellínColombia, fbolanosm@unal.edu.co

4 Ingeniero Eléctrico, Departamento de Energía Eléctrica y Automática, Universidad Nacional de Colombia, Medellín-Colombia, faramirezq@unal.edu.co

5 Ingeniero Eléctrico, Departamento de Energía Eléctrica y Automática, Universidad Nacional de Colombia, Medellín-Colombia, jrcamarillop@unal.edu.co

6 MSc en Ingeniería Eléctrica, Departamento de Electromecánica y Mecatrónica, Facultad de Ingenierías, Instituto Tecnológico Metropolitano, Medellín-Colombia, adrianatrejos@itm.edu.co 


\section{Resumen}

La generación eléctrica híbrida combina las ventajas de las celdas de combustible con sistemas de generación difíciles de predecir, como los fotovoltaicos y eólicos. El principal objetivo en este tipo de sistemas híbridos es minimizar el consumo de hidrógeno reduciendo costos e incrementando la autonomía del sistema. Este articulo propone un algoritmo de optimización, conocido como algoritmo de aprendizaje incremental basado en población, el cual tienen como objetivo maximizar la potencia producida por un generador fotovoltaico. Esta maximización reduce el consumo de hidrógeno combustible del sistema basado en hidrógeno. Adicionalmente, la velocidad de convergencia del algoritmo permite la computación en tiempo real de la mejor configuración para el sistema fotovoltaico, permitiendo una optimización dinámica del consumo de hidrógeno de la celda de combustible. Finalmente, se presenta una validación experimental del sistema considerando 6 paneles fotovoltaicos y una celda de combustible NEXA de 1.2 KW. Esta validación, demuestra la efectividad del algoritmo propuesto para la reducción del consumo de hidrógeno en este tipo de sistemas híbridos.

\section{Palabras clave}

Celda de combustible, reconfiguración sistemas fotovoltaicos, generación híbrida, aprendizaje incremental basado en población.

\section{Abstract}

Hybrid generation combines advantages from fuel cell systems with non-predictable generation approaches, such as photovoltaic and wind generators. In such hybrid systems, it is desirable to minimize as much as possible the fuel consumption, for the sake of reducing costs and increasing the system autonomy. This paper proposes an optimization algorithm, referred to as population-based incremental learning, in order to maximize the produced power of a photovoltaic generator. This maximization reduces the fuel consumption in the hybrid aggregation. Moreover, the algorithm's speed enables the real-time computation of the best configuration for the photovoltaic system, which also optimizes the fuel consumption in the complementary fuel cell system. Finally, a system experimental validation is presented considering 6 photovoltaic modules and a NEXA $1.2 \mathrm{KW}$ fuel cell. Such a validation demonstrates the effectiveness of the proposed algorithm to reduce the hydrogen consumption in these hybrid systems.

\section{Keywords}

fuel cell, reconfiguration of photovoltaic systems, hybrid generation, populationbased incremental learning. 


\section{INTRODUCCIÓN}

Las Celdas de Combustible (CC) producen electricidad a partir de la energía almacenada en reactivos químicos sin utilizar una fase mecánica, de forma similar a las baterías [1]. Por ello, su eficiencia no está limitada por el ciclo de Carnot, lo que permite obtener eficiencias mayores a los generadores basados en combustión interna [2].

Existen múltiples tipos de $\mathrm{CC}$, donde las CC con Membrana de Intercambio Protónico (MIP) son las ampliamente utilizadas en aplicaciones de baja potencia (100 $\mathrm{kW}$ ), debido a su baja temperatura de operación $\left(40^{\circ} \mathrm{C}-80^{\circ} \mathrm{C}\right)$ y su electrolito sólido. Asimismo, Las CC-MIP utilizan reactivos libres de polución [1]-[3]: hidrógeno como fuente de iones $\mathrm{H}^{+}$(combustible) y oxígeno como receptor de los iones $\mathrm{H}^{+}$(oxidante), donde los productos son electricidad, agua y calor de acuerdo con la relación electroquímica presentada en (1) [3].

$4 \mathrm{H}_{2}+2 \mathrm{O}_{2} \rightarrow 4 \mathrm{H}_{2} \mathrm{O}+$ electricidad + calor

Las CC son, entonces, generadores eléctricos con baja huella de carbono, debido a que la generación de energía eléctrica no requiere la combustión de reactivos, por lo que no se produce la emisión de contaminantes a la atmósfera. Esta característica les permite suplir electricidad en aplicaciones aisladas sin contaminar el medio ambiente, de forma similar a los generadores renovables difíciles de predecir, por ejemplo, fotovoltaicos (FV) [2], [3] y eólicos. Debido a que las CC permiten predecir la cantidad de energía que se produce, lo que presenta gran dificultad con los sistemas FV o eólicos [4], las CC son ideales para suplir sistemas críticos tales como protecciones, sistemas de comunicación, entre otros. Adicionalmente, las CC tienen la desventaja de requerir un suministro continuo de combustible, lo que hace necesario almacenar y transportar ese combustible.
Por tanto, algunos autores han propuesto la conexión de generadores adicionales para formar sistemas híbridos con mayor autonomía [1]-[5], es decir, menor consumo de combustible. Esos generadores adicionales deben producir energía sin contaminar y sin requerir combustible, con lo cual los generadores renovables difíciles de predecir son la mejor opción. Dentro de estos, los generadores FV son los más utilizados en la híbridación de CC debido a su reducido mantenimiento y falta de partes móviles. De esta forma, los sistemas híbridos de celdas de combustible y generadores fotovoltaicos (CC-FV) permiten suplir cargas críticas, tales como repetidores de comunicaciones [1], cargadores de vehículos eléctricos [2], protecciones y UPS (Uninterruptible Power Supply) [2]. Este proceso se realiza, además, sin contaminar el medio ambiente y con un consumo de combustible menor en comparación con una $\mathrm{CC}$ aislada, lo que, a su vez, implica una mayor autonomía de servicio.

El principal reto en los sistemas híbridos CC-FV consiste en proveer las condiciones eléctricas requeridas por el bus DC (voltaje, corriente, potencia) al cual están conectados con el menor consumo de combustible posible. Esto comúnmente implica proveer un voltaje regulado al bus, y al mismo tiempo, generar la menor cantidad de energía con la CC. Por tanto, se requiere incrementar la energía producida por el generador FV. Pero, debido a la alta sensibilidad de los sistemas FV a las sombras, esta condición es difícil de cumplir: las sombras parciales afectan severamente la producción energética de sistemas FV [6][9]. Debido a que las sombras parciales son causadas tanto por objetos fijos (árboles, edificios, etc.), los cuales no estaban necesariamente presentes al momento de la instalación del sistema FV, como flotantes (polvo, residuos de pajados, etc.) son imposibles de evitar. Por tanto, para reducir el consumo de combustible en sistemas híbridos CC-FV, se debe mitigar el efecto de las sombras parciales. 
El problema del sombreado parcial se ha abordado en la literatura de dos formas [6]-[9]: controlando la operación de cada módulo FV de forma individual o modificando continuamente la estructura del generador FV. La primera solución, denominada solución distribuida [6], considera el uso de un convertidor DC/DC y un controlador para cada módulo del generador $\mathrm{FV}$, lo que incrementa significativamente el costo, complejidad, peso y área del sistema. En contraste, la segunda solución, denominada reconfiguración [7], solo requiere la adición de una matriz de relés, la cual tiene como objetivo modificar las conexiones eléctricas entre los módulos FV. De esa forma, el sistema FV se reconfigura para reducir el efecto del sombreado parcial e incrementar así la potencia producida. El principal reto en esta técnica consiste en encontrar la configuración eléctrica del sistema FV que provea mayor potencia: debido a que cada módulo FV puede conectarse de múltiples formas, o incluso desconectarse, existen una gran cantidad de posibilidades [7], donde la búsqueda uno-auno (búsqueda exhaustiva o BE) es la opción más comúnmente utilizada. Sin embargo, debido al gran número de configuraciones que se deben evaluar, la reconfiguración basada en $\mathrm{BE}$ es impráctica debido a los tiempos de cálculo excesivamente largos que se requieren.

Como alternativa, este artículo propone el uso del algoritmo de aprendizaje incremental basado en población (AIBP) para buscar la configuración que permita al generador FV proveer la mayor potencia posible en todo momento, lo que a su vez reducirá el consumo de combustible. La organización del artículo es la siguiente: en la primera sección presenta la arquitectura de los sistemas híbridos CC-FV incluyendo el sistema de reconfiguración. La Sección "Sombreado parcial y reconfiguración" describe en detalle el fenómeno de sombreado parcial y el concepto de la reconfiguración eléctrica. La siguiente sección introduce la reconfiguración basada en el algoritmo AIBP y la Sección "Desempeño de la solución basada en AIBP" ilustra sus ventajas con un caso de estudio. Finalmente, las conclusiones se reportan en la última sección.

\section{ARQUITECTURA DE SISTEMAS HÍBRIDOS CC-FV}

Los sistemas híbridos basados en CC tienen una estructura paralela con un bus DC común, lo que permite conectar múltiples fuentes y cargas de forma simple. La Fig. 1 presenta la estructura propuesta para el sistema híbrido, la cual incluye un generador basado en $\mathrm{CC}$, un generador FV, convertidores DC/DC, controladores y el sistema de reconfiguración.

El generador basado en CC está compuesto por diferentes dispositivos: una conexión serie de CC, denominada pila, para proveer voltajes de operación entre 20 $\mathrm{V}$ y $36 \mathrm{~V}$; un compresor de aire que genera el flujo de aire $W_{\text {air }}$ para suplir oxígeno a la CC; y un sistema de almacenamiento de hidrógeno (incluyendo una válvula) para suplir el flujo de combustible $\mathrm{W}_{\mathrm{H} 2}$ a la CC. Igualmente, el generador basado en $\mathrm{CC}$ requiere diferentes sistemas de control. Un primer controlador regula la diferencia de presión entre el ánodo y el cátodo para evitar daños en la membrana [10], y un segundo controlador regula el compresor de aire para evitar la aparición del fenómeno de agotamiento de oxígeno. Este fenómeno ocurre cuando la corriente requerida a la CC no puede suplirse con los reactivos (hidrógeno y oxígeno), por tanto, los electrones y iones $\mathrm{H}^{+}$se extraen de los electrolitos y membrana destruyendo la CC [3][10]. Las relaciones estequiométricas entre los flujos de reactivos y la corriente se reportan en (2), las cuales son obtenidas en [10], donde MO2 y MH2 representan las masas molares del oxígeno e hidrógeno, $\mathrm{n}$ es el número de CC que forman la pila y $\mathrm{F}$ es la constante de Faraday. 


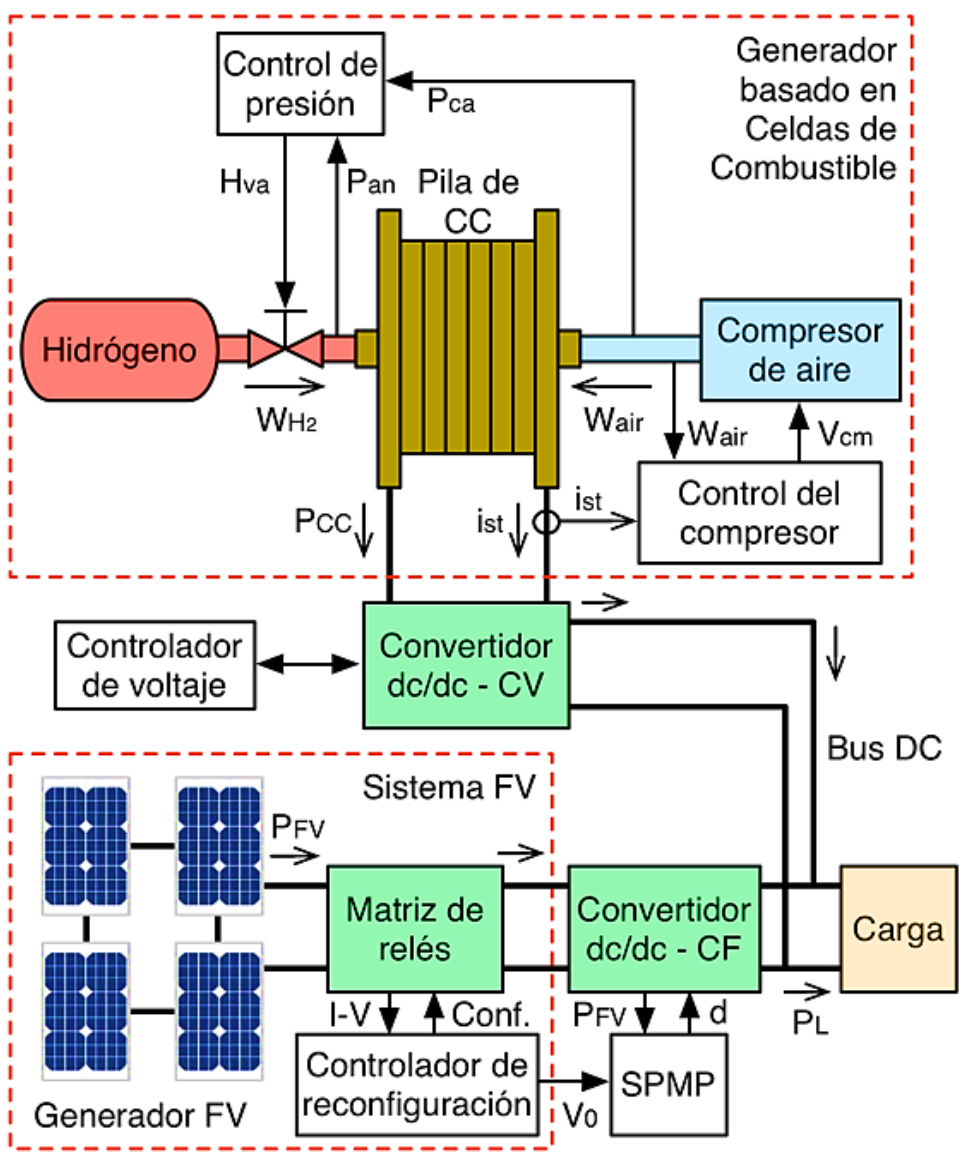

Fig. 1. Sistema híbrido de celdas de combustible y generador fotovoltaico. Fuente: Autores.

A partir de (2) se concluye que el compresor de aire debe suplir un flujo de aire que provea una cantidad de oxígeno mayor a Wo2 para evitar el agotamiento del mismo, esto teniendo en cuenta además que, como se observa en la Fig. 1, el aire se extrae de la atmósfera y por tanto tiene una concentración de oxigeno del $21 \%$ como se reporta en [11], [12]. Finalmente, a partir de (2) se concluye que el consumo de combustible (hidrógeno), depende de la corriente de la CC y no de la apertura de la válvula. Una descripción detallada del comportamiento de la CC y de su modelo se presenta en [10].

$$
W_{O 2}=M_{O 2} \frac{n \cdot i_{s t}}{4 \cdot F} \wedge W_{H 2}=M_{H 2} \frac{n \cdot i_{s t}}{2 \cdot F}
$$

El generador FV se conecta a una matriz de relés, controlada por un algoritmo de reconfiguración, para incrementar la potencia máxima que es posible generar. En todo caso, para garantizar la producción de esa potencia máxima se requiere ubicar el generador FV en su condición óptima de operación [1], denominada punto de máxima potencia (PMP). Debido a que las características de tensión y corriente del PMP no necesariamente corresponden a las condiciones de operación de la carga (tensión y/o corriente), se requiere un convertidor DC/DC (CF) para desacoplar las impedancias del generador FV y del bus DC. De igual forma, debido a que el PMP cambia dependiendo de las condiciones ambientales, se requiere un algoritmo de optimización en-línea, denominado algoritmo de seguimiento del PMP (SPMP), para modificar el ciclo de trabajo d del convertidor CF buscando incrementar la potencia producida PFV. Una descripción detallada de este procedimiento se presenta en [1] y [3]. 
Debido a que la tensión de operación de la CC no necesariamente corresponde con la tensión de operación del bus DC, se requiere un segundo convertidor DC/DC (CV) que desacople los voltajes de la $\mathrm{CC}$ y del bus DC, tal como se ilustra en la Fig. 1. Asimismo, teniendo en cuenta que el convertidor CF se controla para regular la operación del generador FV, es decir sus terminales de entrada, la tensión en sus terminales de salida no se regula. Por lo tanto, el convertidor $\mathrm{CV}$ se debe controlar para regular la tensión del bus DC.

\section{SOMBREADO PARCIAL Y RECONFIGURACIÓN}

Como se observa en la Fig. 1, los generadores FV son conexiones serie-paralelo de módulos FV. En general, los módulos se conectan en serie formando cadenas, las cuales se conectan en paralelo. La Fig. 2 presenta un ejemplo de una cadena de 2 módulos (FV1 y FV2), donde se observa que cada módulo tiene asociado un diodo en anti-paralelo, denominado diodo de bypass. Este diodo evita que el módulo opere en el segundo cuadrante (voltaje negativo) donde disipa potencia, lo que reduce la vida útil del módulo e incluso puede causar su destrucción [8]. La expre- sión (3) describe la relación entre las corrientes de un módulo (iFv) y su tensión $\left(\mathrm{V}_{\mathrm{FV}}\right)$, la cual depende también de los parámetros físicos del módulo: resistencias parásitas serie y paralelo $\left(R_{\mathrm{s}} \mathrm{y} \mathrm{R}_{\mathrm{p}}\right)$, corriente inversa de saturación y voltaje térmico de la juntura ( $\mathrm{I}_{\mathrm{o}} \mathrm{y} \mathrm{V}_{\mathrm{td}}$ ). Adicionalmente, la corriente del módulo depende de la corriente inducida por la radiación solar $\left(\mathrm{I}_{\mathrm{SC}}\right)$.

$i_{F V}=I_{S C}-I_{o} \cdot\left(e^{\frac{R_{S} \cdot i_{F V}+v_{F V}}{v_{t d}}}-1\right)-\frac{R_{S} \cdot i_{F V}+v_{F V}}{R_{p}}$

Por tanto, si en una cadena los módulos producen diferente corriente también generan diferente voltaje. En el ejemplo de la Fig. 2 se observa que el módulo 2 tiene una sombra parcial, lo que hace que su corriente inducida sea menor a la del módulo 1. Asimismo, si se impone a la cadena una corriente ica mayor a Isc2 (corriente inducida al módulo 2), la expresión (3) indica que el módulo 2 opera con un voltaje negativo, i.e. en el segundo cuadrante. Esta condición se previene con el diodo de bypass, el cual se activa cuando la tensión del módulo se hace negativa, permitiendo el paso del exceso de corriente $i_{d b 2}=i_{c a}-I_{\mathrm{SC} 2}$. De esta forma, cuando el diodo de bypass se activa el módulo no produce potencia, pero se evita su degradación.

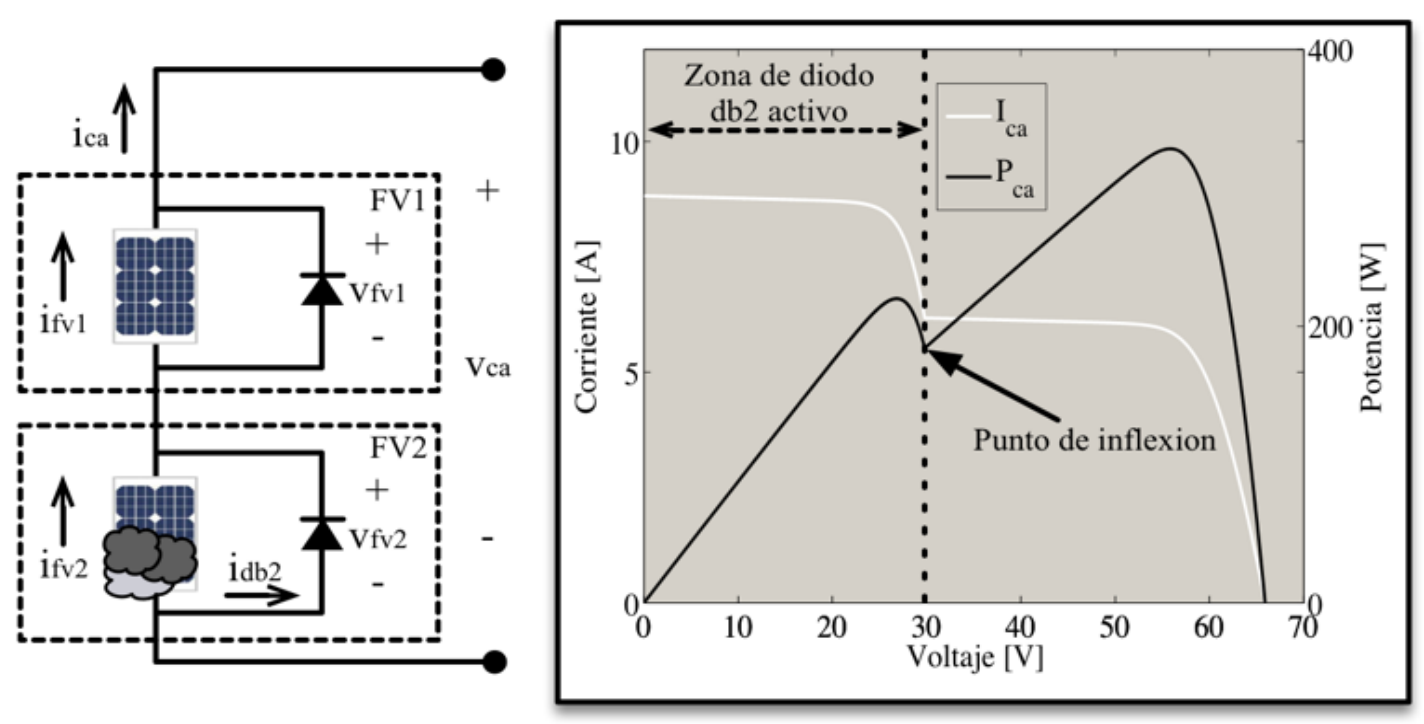

Fig. 2. Efecto de sombreado parcial en una cadena de módulos FV. Fuente: Autores. 


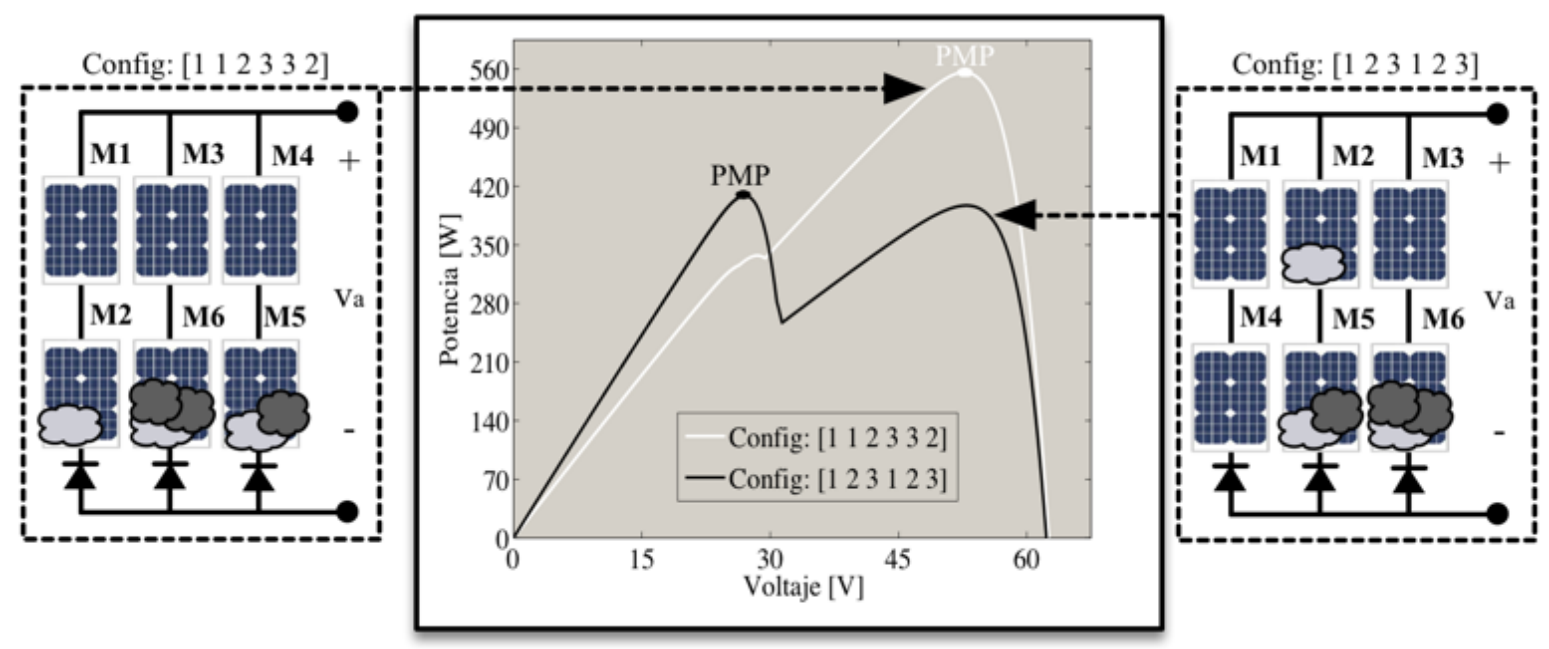

Fig. 3. Efecto de la reconfiguración eléctrica de un generador FV. Fuente: Autores.

El comportamiento anteriormente descrito genera que algunos módulos se activen o desactiven dependiendo de las sombras y de la corriente impuesta a la cadena. Estas condiciones se ilustran en la parte derecha de la Fig. 2, la cual presenta la simulación de la cadena formada por módulos Kyocera KD220: el primer módulo (completamente irradiado) tiene una radiación de $1000 \mathrm{~W} / \mathrm{m}^{2}$ y el segundo módulo (sombreado parcialmente) tiene una radiación de $700 \mathrm{~W} / \mathrm{m}^{2}$. De esta forma, si la corriente de la cadena es mayor a $6 \mathrm{~A}$ el diodo de bypass del módulo 2 (db2) se activa, dejando al módulo 1 como única fuente de energía. En contraste, si la corriente de la cadena es menor a 6 A los dos módulos producen energía. Este punto límite, $6 \mathrm{~A}$ para la Fig. 2, cambia dependiendo de las condiciones de sombreado y de la estructura eléctrica del generador $\mathrm{FV}$.

La Fig. 3 ilustra el efecto de modificar las conexiones eléctricas de un generador FV con sombreado parcial, i.e. reconfiguración eléctrica: el generador está formato por 6 módulos M1 a M6, donde M1, M3 y M4 no están sombreados, M2 tiene sombreado un $25 \%$ del área, M5 tiene sombreado un $50 \%$ del área y M6 tiene un 75 $\%$ del área sombreada. Bajo estas condiciones, la configuración [1 11223 2 3 2] (M1 y M2 en la primera cadena, M3 y M6 en la se- gunda cadena y M4 y M5 en la tercera cadena) tiene un PMP que provee $415 \mathrm{~W}$. Sin embargo, estos mismos módulos configurados en [ $\left[\begin{array}{llllll}1 & 2 & 3 & 1 & 2 & 3\end{array}\right]$ generan un PMP que provee $558 \mathrm{~W}$; es decir, la producción del generador FV se incrementa en $34.4 \%$ al modificar las conexiones eléctricas entre los módulos FV.

Debido a que las condiciones de sombreado cambian continua e impredeciblemente, se requiere reconfigurar el generador FV continuamente. Para ello se utiliza la matriz de relés que interconecta el generador FV con el convertidor CF en la Fig. 1. La Fig. 4 presenta una descripción más detallada de la matriz de relés: cada módulo cuenta con $2 \mathrm{C}$ relés, donde $\mathrm{C}$ representa el número máximo de cadenas del generador. De esta forma, cada módulo puede conectarse a cualquier cadena, o incluso desconectarse del generador. La salida de la matriz corresponde a las terminales del generador, las cuales se conectan al convertidor CF para realizar el SPMP.

Por tanto, la reconfiguración del generador FV requiere detectar la configuración eléctrica que provee mayor potencia. Esta tarea se realiza comúnmente utilizando el algoritmo BE, el cual requiere evaluar cada una de las opciones [7]. Teniendo en cuenta que el número de configuraciones posibles es $(\mathrm{N}+1)^{\mathrm{N}}$ donde $\mathrm{N}$ representa el 
número de módulos [6], evaluar cada posible configuración requiere tiempos de procesamiento imprácticamente largos. Por ejemplo, para $\mathrm{N}=6$, como en las Figs. $3 \mathrm{y}$ 4, existen 117649 posibles configuraciones, cuya evaluación tarda 37 minutos en un computador personal. Es decir, la reconfiguración ocurre 37 minutos después de medir las condiciones de operación, tiempo durante el cual las condiciones pueden cambiar significativamente, y por tanto la reconfiguración es inefectiva o incluso negativa para la potencia producida.

\section{RECONFIGURACIÓN BASADA EN EL ALGORITMO AIBP}

Con el objetivo de reconfigurar el generador $\mathrm{FV}$ en un tiempo corto, de forma que las condiciones ambientales no cambien significativamente y así obtener una reconfiguración efectiva, este artículo propone utilizar el algoritmo de aprendizaje incremental basado en población, el cual no requiere evaluar todas las posibles soluciones.

El algoritmo AIBP utiliza una matriz, como la presentada en la Fig. 5, para almacenar las posibles soluciones y su probabilidad de éxito. El AIBP es similar a los algoritmos genéticos tradicionales, pero debido a que poblaciones enteras evolucionan (en lugar de individuos) es más simple de implementar. El algoritmo inicia con todas las probabilidades iguales para no sesgar la búsqueda; luego, en cada iteración se crean nuevas poblaciones y se actualizan las probabilidades de cada solución, de forma que el AIBP converge a la solución óptima. Una descripción detallada del algoritmo se presenta en [13] y [14], donde los pasos básicos del mismo son:

1. Generar una población a partir del genotipo de la población (matriz de probabilidades, e.g. Fig. 5).

2. Evaluar la función de costo en cada miembro para priorizar las soluciones más viables.
3. Actualizar el genotipo de la población a partir de los resultados del paso 2.

4. Repetir pasos del 1 al 3 hasta alcanzar el límite de convergencia.

En el caso particular de la reconfiguración del generador FV, la matriz de probabilidades (Fig. 5) se describe en términos de las posibles estructuras de cada cadena: las filas corresponden a las cadenas $(\mathrm{Ca} 0$ representa desconexión) y las columnas corresponden a los módulos. Por tanto, la posición $\mathrm{P}(\mathrm{i}, \mathrm{j})$ describe la probabilidad que el módulo j sea parte de la cadena $\mathrm{i}$.

Una probabilidad alta implica que la conexión asociada incrementa la potencia generada. En todo caso, es imposible para un módulo estar conectado a más de una cadena, por tanto, las probabilidades de cada columna, i.e. asociadas a un mismo módulo, deben sumar 1 (100\%) como se describe en (4). Igualmente, las probabilidades iniciales se definen de acuerdo con (5) para garantizar que todas las configuraciones iniciales tienen la misma probabilidad en el inicio de la optimización.

$\sum_{i=0}^{N} P(i, j)=1, \forall 1 \leq j \leq N$
$\left.P(i, j)\right|_{0}=\frac{1}{N+1}, \forall 1 \leq j \leq N \wedge \forall 0 \leq i \leq N$

El algoritmo propuesto utiliza la función de costo descrita en (6), la cual está basada en una regla de aprendizaje Hebbian [13], donde $\mathrm{P}(\mathrm{i}, \mathrm{j})$ ant representa la probabilidad inmediatamente anterior de la posición (i,j). El valor de $\mathrm{k}$ corresponde a la cadena en la cual el módulo tiene mayor probabilidad $(0 \leq \mathrm{k} \leq \mathrm{N})$, por tanto, cuando la probabilidad en $\mathrm{k}$ se incrementa las otras probabilidades decrecen para cumplir (4). Finalmente, LR parametriza la velocidad de convergencia del algoritmo sin incurrir en pasos excesivamente largos que lleven a un ciclo límite [13]-[15].

$P(i, j)=\left\{\begin{array}{c}P(i, j)_{a n t}+\left(1-P(i, j)_{a n t}\right) \cdot L R, \text { si } i=k \\ (1-P(k, j)) \cdot \frac{P(i, j)_{\text {ant }}}{1-P(i, j)_{a n t}}, \text { si } i \neq k\end{array}\right.$ 


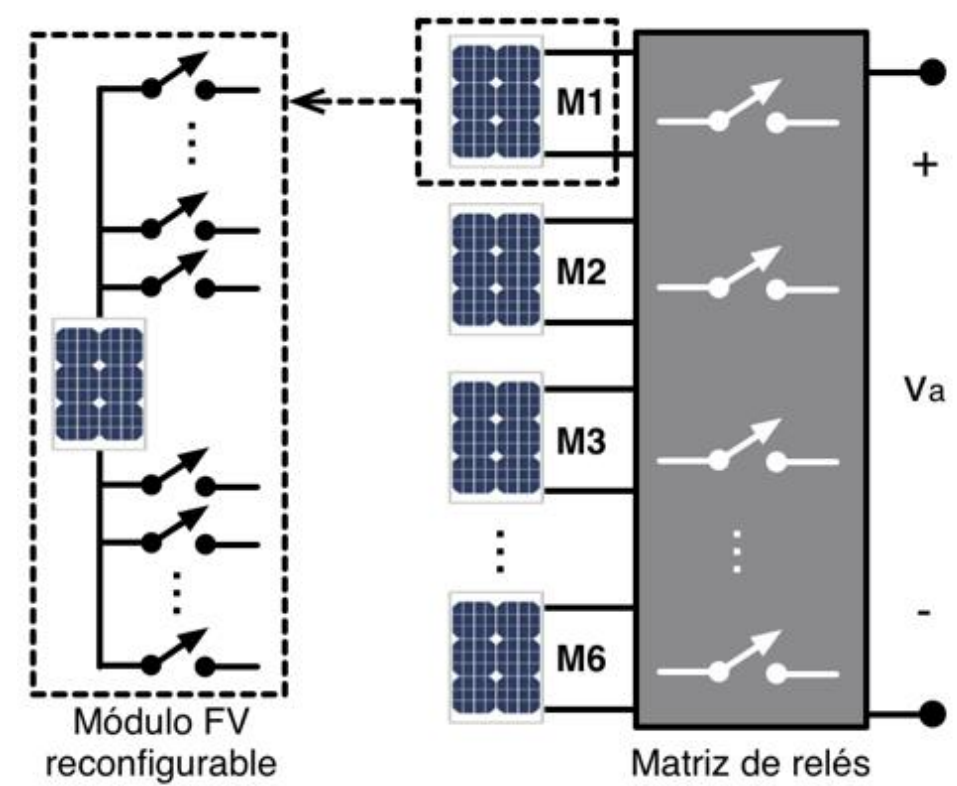

Fig. 4. Matriz de relés para reconfiguración de generadores FV. Fuente: Autores.

\begin{tabular}{|c|c|c|c|c|c|c|}
\hline & M1 & M2 & M3 & M4 & M5 & M6 \\
\hline $\mathrm{Ca} 0$ & $\mathrm{P}(0,1)$ & $\mathrm{P}(0,2)$ & $\cdots$ & $\cdots$ & $\cdots$ & $\mathrm{P}(0,6)$ \\
\hline $\mathrm{Ca} 1$ & $\mathrm{P}(1,1)$ & & & & & $:$ \\
\hline $\mathrm{Ca} 2$ & : & & & & & $:$ \\
\hline $\mathrm{Ca} 3$ & $:$ & & & & & $:$ \\
\hline $\mathrm{Ca} 4$ & : & & & & & $:$ \\
\hline $\mathrm{Ca} 5$ & : & & & & & $:$ \\
\hline $\mathrm{Ca} 6$ & $\mathrm{P}(6,1)$ & $\cdots$ & $\cdots$ & $\cdots$ & $\cdots$ & $\mathrm{P}(6,6)$ \\
\hline
\end{tabular}

Fig. 5. Genotipo del algoritmo AIBP: matriz de probabilidades. Fuente: Autores.

Para definir la suficiencia de una solución, el AIBP utiliza el concepto de entropía descrito en (7), el cual provee una valoración de la distribución de las probabilidades en la matriz: E es máximo cuando las probabilidades son iguales, y converge a cero cuando las probabilidades se concentran alrededor de una solución particular. Para proveer un valor estándar, se utiliza el concepto de entropía normalizada, la cual corresponde a $\mathrm{E}_{\mathrm{N}}=\mathrm{E} / \mathrm{E}_{\mathrm{MAX}}$, donde $\mathrm{E}_{\mathrm{MAX}}=\mathrm{N} \cdot(\mathrm{N}+1)$ corresponde al valor má- ximo de E, i.e. cuando todas las probabilidades son iguales. Finalmente, el algoritmo se detiene cuando el genotipo del sistema presenta una entropía normalizada menor a un valor de tolerancia $\mathrm{E}_{\mathrm{Tol}}$. $\mathrm{La}$ correcta selección de $\mathrm{E}_{\mathrm{Tol}}$ garantiza una solución que provea alta potencia sin evaluar todas las posibles configuraciones.

$$
E=\sum_{i=0}^{N} \sum_{j=1}^{N} P(i, j) \cdot \log _{10}[P(i, j)], 0<E<1
$$




\section{DESEMPEÑO DE LA SOLUCIÓN BASADA EN AIBP}

La efectividad de la arquitectura híbrida con reconfiguración se validó bajo las condiciones de irradiación solar presentes en Colombia central, considerando un perfil dinámico de sobras moviéndose horizontalmente a través del generador FV. La Fig. 6 ilustra el perfil de sombreado, en el cual a las 8:00 la primera cadena (M1 y M4) comienza a sufrir sombreado parcial. La sombra se mueve de forma que a las 12:00 la segunda cadena (M2 y M5) también se ve afectada. Finalmente, a las 16:00 la tercera cadena (M3 y M6) también sufre sombreado. La parte superior de la Fig. 6 describe el porcentaje del área sombrada para cada módulo en las diferentes horas del día.

Asimismo, los convertidores DC/DC de la arquitectura híbrida (Fig. 1) utilizados en las pruebas experimentales tienen una eficiencia promedio de $90 \%$. La Fig. 7 presenta el prototipo experimental, el cual está compuesto por un emulador del sistema FV que incluye el generador PV y la matriz de relés, el convertidor DC/DC CF, y un DSP que ejecuta el SPMP y el controlador de reconfiguración. La figura también presenta la CC NEXA utilizada para calcular el consumo de combustible a partir de los perfiles de potencia de la siguiente forma: los perfiles de potencia, medidos en el prototipito utilizando la tarjeta de control, se imponen a un modelo de pila de combustible, reportado en [10] e implementado en Simulink, el cual calcula el consumo de combustible correspondiente a ese perfil de potencia.

Con el objetivo de evaluar la velocidad de cálculo del enfoque AIBP, se efectuó una serie de pruebas para medir el tiempo de convergencia del algoritmo con diferentes tamaños del generador FV. Los resultados se presentan en la Fig. 8, donde se observa que el tiempo de convergencia del algoritmo AIBP está por debajo de los 2 minutos para generadores con menos de 13 módulos. En particular, para el generador con 6 módulos considerado para la validación (Fig. 6), el algoritmo AIBP tarda 13 segundos en ejecutar la reconfiguración en lugar de los 37 minutos requeridos por el algoritmo BE. Por tanto, el algoritmo AIBP es viable para reconfiguración en tiempo real, lo que no es posible con el algoritmo BE.

Teniendo el algoritmo AIBP correctamente parametrizado para 6 módulos, se ejecutaron dos experimentos. El primero de ellos considera el generador FV estático, en el cual el SPMP controla el convertidor CF para buscar la máxima potencia disponible en la configuración por defecto [ $\left[\begin{array}{llllll}1 & 2 & 3 & 1 & 2 & 3\end{array}\right]$. El segundo experimento, considera la reconfiguración basada en AIBP, en la cual la matriz de relés modifica la configuración para garantizar la condición con mayor potencia disponible; y al mismo tiempo el SPMP controla el convertidor $\mathrm{CF}$ para buscar la máxima potencia. En este segundo caso, el controlador de reconfiguración, además, envía la posición del PMP al SPMP, con lo cual el tiempo requerido para alcanzar la máxima producción de potencia es mucho menor con respecto al primero caso. Este comportamiento se verifica en la Fig. 9, la cual presenta la potencia producida en los dos experimentos para condiciones cambiantes de irradiación y sombreado, donde las sombras se modifican de acuerdo con las condiciones presentadas en la Fig. 6. Estos resultados demuestran que el sistema de reconfiguración (PFV-Basado en AIBP) produce mayor energía en comparación con el generador fotovoltaico tradicional (PFV-Estático). De igual manera, se verifica que la solución basada en AIBP converge igual o más rápido a la potencia óptima, por ejemplo, en la condición $\mathrm{S}=$ $150 \mathrm{~W} / \mathrm{m}^{2}$. 


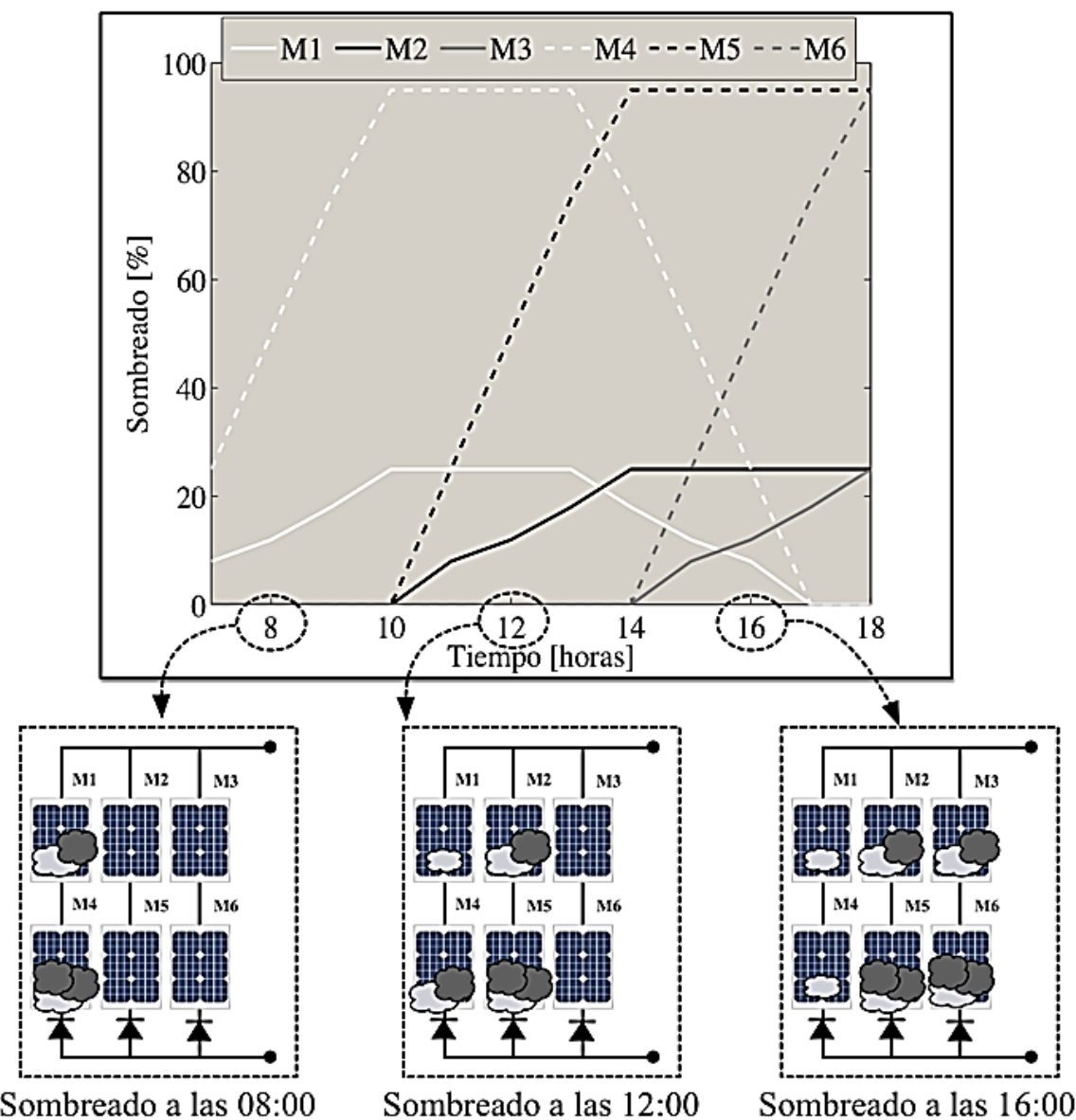

Fig. 6. Perfil dinámico de sombras utilizado para validación. Fuente: Autores.

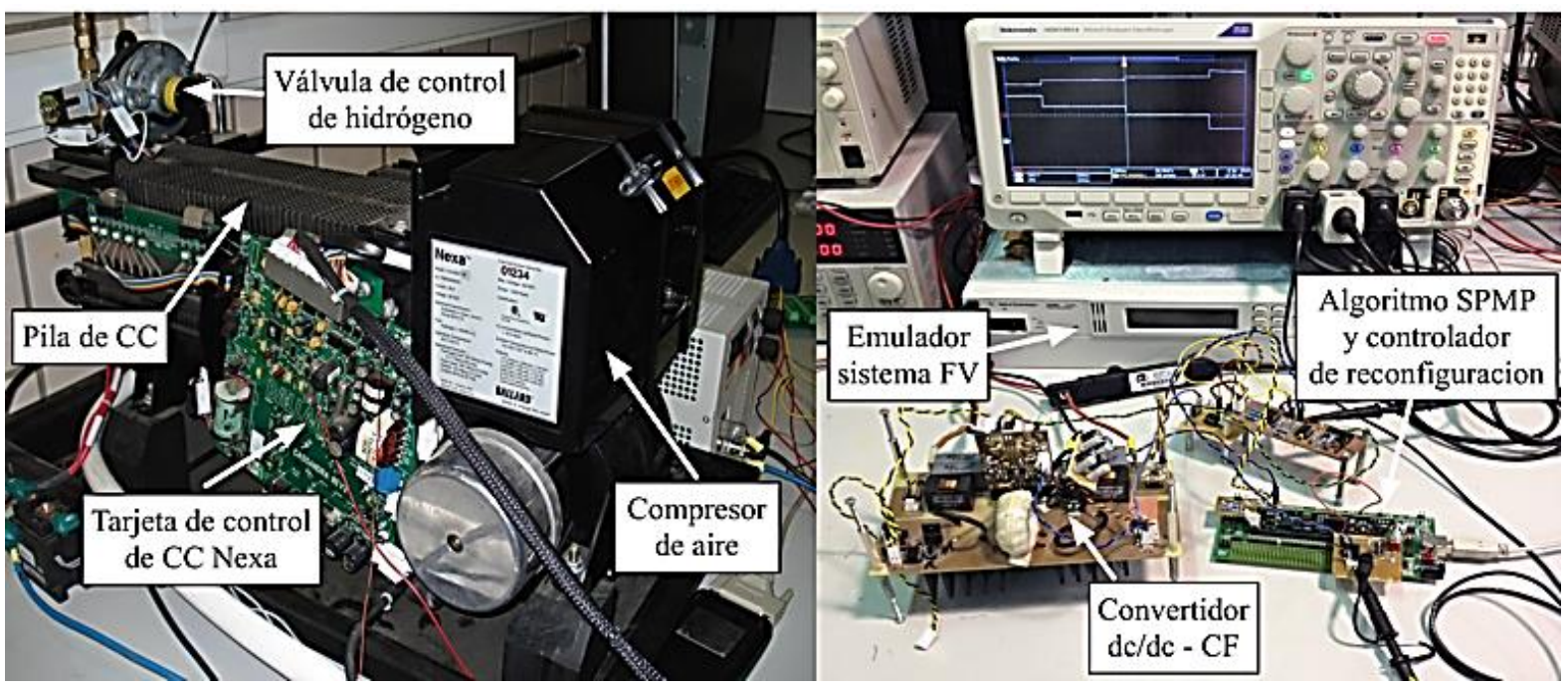

Fig. 7. Prototipo experimental. Fuente: Autores. 
Reconfiguración de paneles fotovoltaicos para reducción del consumo de hidrógeno en las celdas de combustible de sistemas híbridos

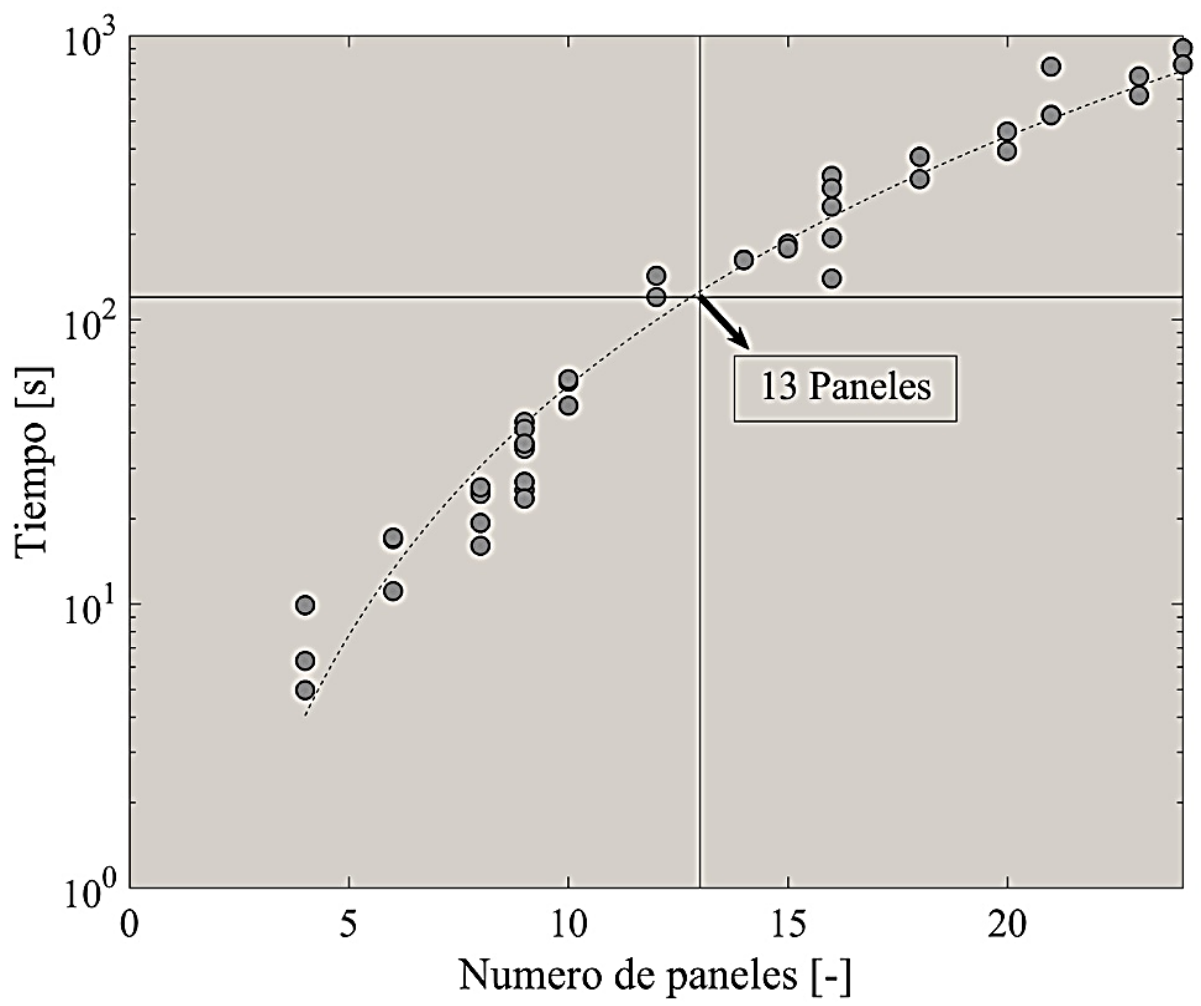

Fig. 8. Tiempos de procesamiento del algoritmo AIBP. Fuente: Autores.

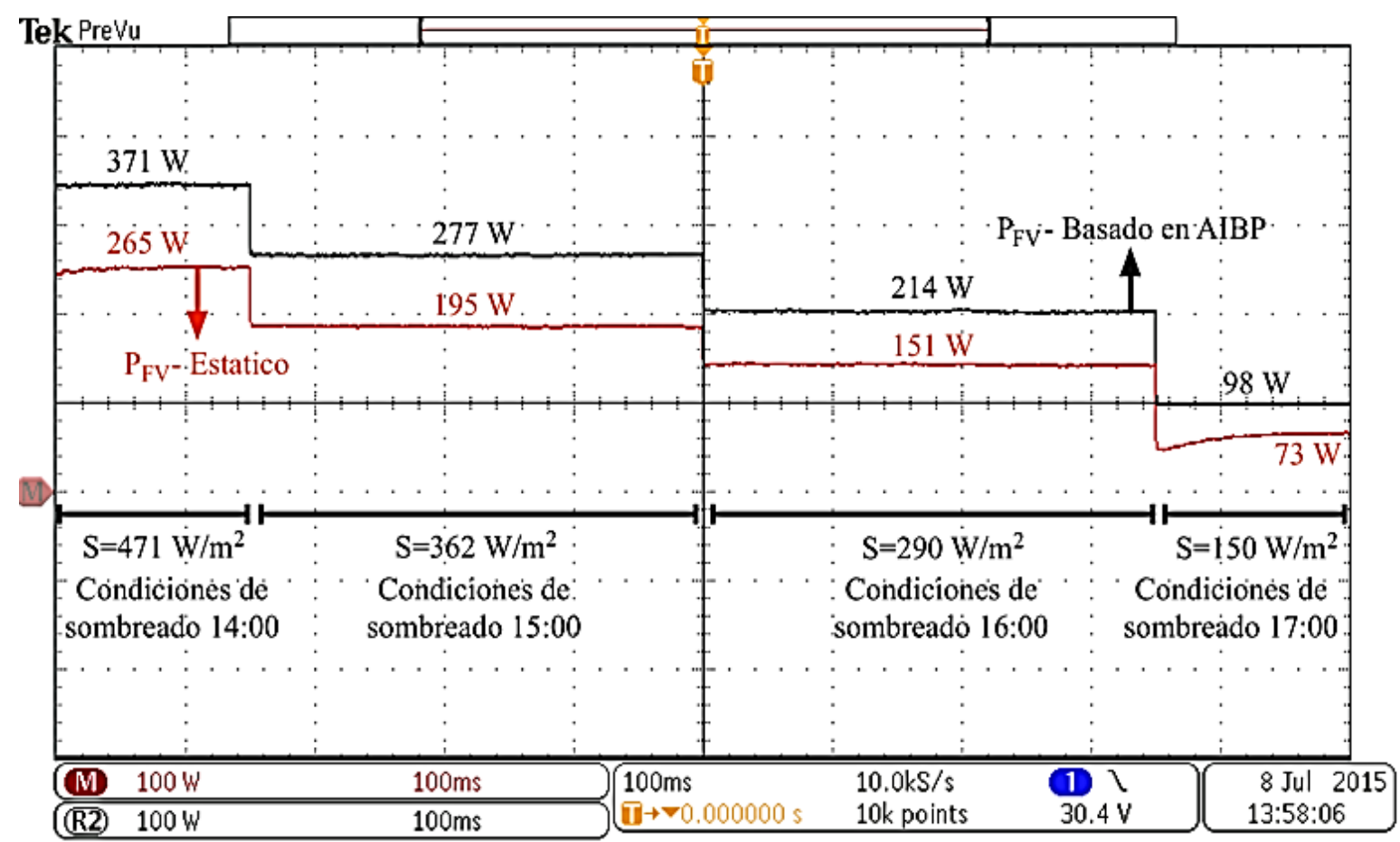

Fig. 9. Comparación experimental de la potencia producida por el sistema estático y el sistema basado en AIBP. Fuente: Autores. 




Fig. 10. Consumo de combustible del sistema híbrido en un día típico de Colombia central usando las soluciones AIBP y estática. Fuente: Autores.

Finalmente, utilizando el sistema experimental se reprodujo el comportamiento del sistema durante un día típico en Colombia central, cuyo perfil de radiación solar se presenta en la Fig. 10. Adicionalmente, los experimentos consideraron el perfil dinámico de sombras presentado en la Fig. 6. A partir de los perfiles de potencia del generador FV, se calculó el consumo de hidrógeno requerido para suplir la carga en los dos casos evaluados utilizando el modelo de la NEXA [10]. Los resultados, presentados en la Fig. 10, verifican que la solución basada en AIBP reduce significativamente el consumo de hidrógeno. Adicionalmente, se puede calcular la eficiencia del sistema para ambos escenarios frente a una instalación con CC como único generador de energía eléctrica. A partir de la relación $E_{F}=\frac{H_{C C}-H}{H_{C C}}$ se puede calcular la eficiencia de dicho sistema EF, donde, HCC representa el hidrógeno consumido por un sistema basado solo en la generación de energía con la CC y H representa el hidrógeno consumido en ambos escenarios (Es- tático o Basado en AIBP). Para el primer caso donde no se tiene en cuenta la reconfiguración de los módulos la eficiencia del sistema frente a una instalación de CC como única fuente es del $18 \%$. Por el contrario, la eficiencia del sistema teniendo en cuenta la reconfiguración es del 26\%. Esto demuestra que el sistema basado en una configuración híbrida es viable para la reducción del consumo de hidrógeno, como también, el concepto de reconfiguración demuestra una optimización del hidrógeno con respecto a una instalación estática de paneles fotovoltaicos.

\section{CONCLUSIONES}

Se propuso y validó un esquema de reconfiguración dinámica para un sistema de generación híbrido, basado en celdas de combustible y módulos fotovoltaicos. Dicho esquema está basado en el algoritmo de aprendizaje incremental basado en población (AIBP), cuya velocidad de convergen- 
cia permite ejecutar el cálculo en tiempo real de la configuración óptima para la conexión de los módulos fotovoltaicos. Los resultados obtenidos demuestran el desempeño superior del algoritmo AIBP con respecto al enfoque tradicional, basado en búsquedas exhaustivas. La optimización efectuada por el algoritmo AIBP maximiza la potencia producida por el sistema fotovoltaico, reduciendo a su vez el consumo de combustible, lo que representa una reducción de los costos de operación del sistema y un incremento en la autonomía del mismo. De igual manera, se presentó un cálculo de la eficiencia del sistema comparando los casos donde se tiene en cuenta el reconfigurador y donde no está presente, con respecto a un sistema basado en $\mathrm{CC}$ como única fuente generadora de energía. El cálculo de la eficiencia valida que este tipo de soluciones son viables para ser implementadas como estrategia de optimización del hidrógeno consumido por la CC.

Por otra parte, debido a que la celda de combustible no es reversible, se requiere de baterías o supercondensadores para almacenar el exceso de energía producido cuando la carga tiene bajo consumo, tal y como se reporta en múltiples implementaciones híbridas reportadas en la literatura [16][18]. Este sistema de almacenamiento permite suplir transitorios de cargas DC, por ejemplo, el encendido de un sistema de iluminación y la puesta en marcha de un sistema de comunicaciones. Asimismo, como se reporta en [17], [18], un sistema híbrido $\mathrm{CC} / \mathrm{FV}$ con baterías puede suplir cargas $\mathrm{AC}$, ya que la componente $\mathrm{AC}$ de la potencia requerida por el inversor se suple a partir de la batería, y el sistema híbrido suple el consumo promedio de potencia.

A nivel comercial, esta solución puede implementarse en sistemas embebidos, tales como DSP o FPGA, para su difusión comercial. En todo caso, el requerimiento de tiempos de cálculo cortos hace necesario explorar, en trabajos futuros, la implementación de esta solución en sistemas de procesamiento en paralelo, lo que permitirá la reconfiguración en tiempo real de sistemas híbridos de gran tamaño.

Asimismo, es de notar que, debido al decremento en la potencia producido por sistemas fotovoltaicos bajo sombreado parcial, el forecasting clásico para sistemas FV con conexiones fijas presentará una sobre estimación de energía. Es por eso que existen nuevos métodos para la estimación de la potencia producida por sistemas FV con sombreado parcial, por ejemplo, las soluciones reportadas en [19], [20]. Por lo tanto, un campo abierto de investigación consiste en incluir esos métodos dentro de los algoritmos clásicos de forecasting para incrementar su precisión, incluyendo además el efecto de la reconfiguración de las conexiones entre los paneles $\mathrm{FV}$, lo que permitirá proveer un análisis de viabilidad económica más realista de esta nueva solución.

\section{AGRADECIMIENTOS}

Este trabajo fue soportado por el grupo de Automática, Electrónica y Ciencias Computacionales del Instituto Tecnológico Metropolitano, el Laboratorio de Calidad del Aire - CALAIRE, la Universidad Nacional de Colombia y Colciencias dentro del proyecto MicroRENIZ-25439 (Código 1118669-46197) y la beca doctoral 2012-567.

\section{REFERENCIAS}

[1] N. Bigdeli, "Optimal management of hybrid PV/fuel cell/battery power system: A comparison of optimal hybrid approaches," Renew. Sustain. Energy Rev., vol. 42, pp. 377-393, Feb. 2015.

[2] M. Patterson, N. F. Macia, and A. M. Kannan, "Hybrid Microgrid Model Based on Solar Photovoltaic Battery Fuel Cell System for Intermittent Load Applications," IEEE Trans. Energy Convers., vol. 30, no. 1, pp. 359-366, Mar. 2015.

[3] M. S. Behzadi and M. Niasati, "Comparative performance analysis of a hybrid PV/FC/battery stand-alone system using 
Reconfiguración de paneles fotovoltaicos para reducción del consumo de hidrógeno en las celdas de combustible de sistemas híbridos

different power management strategies and sizing approaches," Int. J. Hydrogen Energy, vol. 40, no. 1, pp. 538-548, Jan. 2015.

[4] C. Wan, J. Zhao, Y. Song, Z. Xu, J. Lin, and $\mathrm{Z}$. Hu, "Photovoltaic and solar power forecasting for smart grid energy management," CSEE J. Power Energy Syst., vol. 1 , no. 4 , pp. $38-46$, Dec. 2015.

[5] N. Bizon, M. Oproescu, and M. Raceanu, "Efficient energy control strategies for a Standalone Renewable/Fuel Cell Hybrid Power Source," Energy Convers. Manag., vol. 90, pp. 93-110, Jan. 2015.

[6] E. Romero-Cadaval, G. Spagnuolo, L. G. Franquelo, C. A. Ramos-Paja, T. Suntio, and W. M. Xiao, "Grid-Connected Photovoltaic Generation Plants: Components and Operation," IEEE Ind. Electron. Mag., vol. 7, no. 3, pp. 6-20, Sep. 2013.

[7] J. D. Bastidas-Rodríguez, C. A. Ramos-Paja, and A. J. Saavedra-Montes, "Reconfiguration analysis of photovoltaic arrays based on parameters estimation," Simul. Model. Pract. Theory, vol. 35, pp. 50-68, Jun. 2013.

[8] G. Petrone and C. A. Ramos-Paja, "Modeling of photovoltaic fields in mismatched conditions for energy yield evaluations," Electr. Power Syst. Res., vol. 81, no. 4, pp. 1003-1013, Apr. 2011.

[9] J. D. Bastidas, E. Franco, G. Petrone, C. A. Ramos-Paja, and G. Spagnuolo, "A model of photovoltaic fields in mismatching conditions featuring an improved calculation speed," Electr. Power Syst. Res., vol. 96, pp. 81-90, Mar. 2013.

[10] C. A. Ramos-Paja, R. Giral, L. MartínezSalamero, J. Romano, A. Romero, and G. Spagnuolo, "A PEM Fuel-Cell Model Featuring Oxygen-Excess-Ratio Estimation and Power-Electronics Interaction," IEEE Trans. Ind. Electron., vol. 57, no. 6, pp. 1914-1924, Jun. 2010.

[11] P. Bělohradský, P. Skryja, and I. Hudák, "Experimental study on the influence of oxygen content in the combustion air on the combustion characteristics," Energy, vol. 75, pp. 116-126, Oct. 2014.
[12] T. Hordé, P. Achard, and R. Metkemeijer, "PEMFC application for aviation: Experimental and numerical study of sensitivity to altitude," Int. J. Hydrogen Energy, vol. 37, no. 14, pp. 10818-10829, Jul. 2012.

[13] K. A. Folly and G. K. Venayagamoorthy, "Power system controller design using multipopulation PBIL," in 2013 IEEE Computational Intelligence Applications in Smart Grid (CIASG), 2013, pp. 37-43.

[14] B. N. Bolanos F., Aedo J.E., Rivera F., "Mapping and scheduling in heterogeneous NoC through population-based incremental learning," J. Univers. Comput. Sci., vol. 18, no. 7, pp. 901-916, 2012.

[15] B. N. Bolanos F., Aedo J.E., Rivera F., "Comparison of learning rules for adaptive population-based incremental learning algorithms," in Proc. Int. Conf. Artif. Intel., 2012, pp. 244-251.

[16] M. F. Ezzat and I. Dincer, "Development, analysis and assessment of a fuel cell and solar photovoltaic system powered vehicle," Energy Convers. Manag., vol. 129, pp. 284292, Dec. 2016.

[17] H. Fathabadi, "Novel highly accurate universal maximum power point tracker for maximum power extraction from hybrid fuel cell/photovoltaic/wind power generation systems," Energy, vol. 116, pp. 402-416, Dec. 2016.

[18] I. Tegani, A. Aboubou, M. Y. Ayad, R. Saadi, M. Becherif, M. Bahri, M. Benaouadj, and O. Kraa, "Experimental validation of differential flatness-based control applied to stand alone using photovoltaic/fuel cell/battery hybrid power sources," Int. J. Hydrogen Energy, vol. 42, no. 2, pp. 15101517, Jan. 2017.

[19] D. Picault, B. Raison, S. Bacha, J. de la Casa, and J. Aguilera, "Forecasting photovoltaic array power production subject to mismatch losses," Sol. Energy, vol. 84, no. 7, pp. 1301-1309, Jul. 2010.

[20] S. Pareek and R. Dahiya, "Enhanced power generation of partial shaded photovoltaic fields by forecasting the interconnection of modules," Energy, vol. 95, pp. 561-572, Jan. 2016 . 